\title{
RXTE observations of single pulses of PSR B0531+21
}

\section{Flux variations}

\author{
M. Vivekanand ${ }^{\star}$ \\ National Center for Radio Astrophysics, TIFR, Pune University Campus, PO Box 3, Ganeshkhind, \\ Pune 411007, India
}

Received 8 November 2000 / Accepted 2 May 2001

\begin{abstract}
This article is the first in the series that analyze about 1.87 million periods of PSR B0531+21 (Crab pulsar), observed by the PCA detector aboard the RXTE X-ray observatory. The Crab pulsar's X-ray light curve shows little variation over time scales ranging from days to a period (33.46 milliseconds). The standard deviation of its X-ray flux variation is $\approx 0.7 \%$ of its mean value, which is negligible compared to its radio flux variations. The phase resolved power spectrum of pulse to pulse X-ray flux variation shows no spectral feature; an upper limit to the peak of any possible broad spectral feature is $0.06 \%$ of the mean power. The X-ray fluxes in the two components of its integrated profile are unrelated to each other; their linear correlation coefficient is $0.0004 \pm 0.0010$. "Giant pulses" that are routinely seen at radio wavelengths are absent here. This work sets very strong constraints on the connection (if any) between the flux variations at radio and X-ray energies, for example due to variation in the degree of coherence of the basic emitters. Its phase resolved X-ray flux variation shows a weak correlation with the integrated profile. If confirmed, this might be an important clue to understanding the X-ray emission mechanism of Crab pulsar.
\end{abstract}

Key words. pulsars - individual: PSR B0531+21 - X-ray: stars

\section{Introduction}

The intrinsic temporal flux variations of pulsars are probably an important clue to understanding their emission mechanism at any wavelength. In fact, the flux variations at high energies (optical, X-ray, $\gamma$-ray) might provide important constraints to the emission mechanism at radio wavelengths also (Cheng et al. 1986b; Kawai et al. 1991; Lundgren et al. 1995; Moffet \& Hankins 1996; Patt et al. 1999). Patt et al. (1999) searched for period to period flux variations in about 105000 periods of Crab pulsar data at X-ray energies (in the range 1 to 10 kilo electron volts $(\mathrm{KeV}))$, with a time resolution of 100 microseconds $(\mu \mathrm{s})$, obtained by the PCA detector aboard the RXTE X-ray observatory. This work reports the results of analyzing 1868112 periods of Crab pulsar from the same instrument, with a time resolution of $3.815 \mu \mathrm{s}$, in the energy range 13.3 to $58.4 \mathrm{KeV}$.

The RXTE data archive was searched using the XDF tool, for public data acquired by the Proportional Counter Array (PCA). A uniform set of 23 data files were found observed during August/September 1996, their ObsId numbers ranging from 10203-01-01-00 to 10203-01-03-01. They were obtained in the EVENT mode (XTE_SE), combining events from all five Proportional Counter Units $\left(\mathrm{D}\left[0^{\sim} 4\right]\right)$,

* e-mail: vivek@ncra.tifr.res.in and also from both halves of all three Xenon anode layers

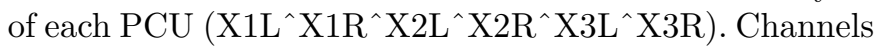
50 to 249 of the PCA were also combined, which corresponds to the energy range 13.3 to $58.4 \mathrm{KeV}$.

The first phase of data analysis used the FTOOLS software. First, the Good Time Intervals (GTI) were obtained for each data file by using the MAKETIME tool on the corresponding XTE filter file; the selection criteria were (a) pointing OFFSET less than $0.02^{\circ}$, (b) elevation (ELV) greater than $10^{\circ}$, and (c) all five PCUs to be switched on. Next, the GTI extension of each data file was edited to insert the above GTI values. Then the FSELECT tool was run to filter out data outside these time ranges. Next the SEFILTER tool was run with the M[1]\{1\} option (without bypassing the FSELECT tool) to retain only the valid photon events. Then the FXBARY tool was run using the orbit file for that day, to convert the arrival times of photons from the Terrestrial Time system (TT) to the solar system barycenter system (TDB). Penultimately, the SEEXTRCT tool was used to obtain the light curve for each file, in time intervals of 1.010895 millisecond (ms), which is 265 times the basic time resolution of the data. Finally, the PCADTLC tool was used to correct the light curve for dead time of the PCA; before this the corresponding Standard 1 files were also converted to the TDB system for consistency. 


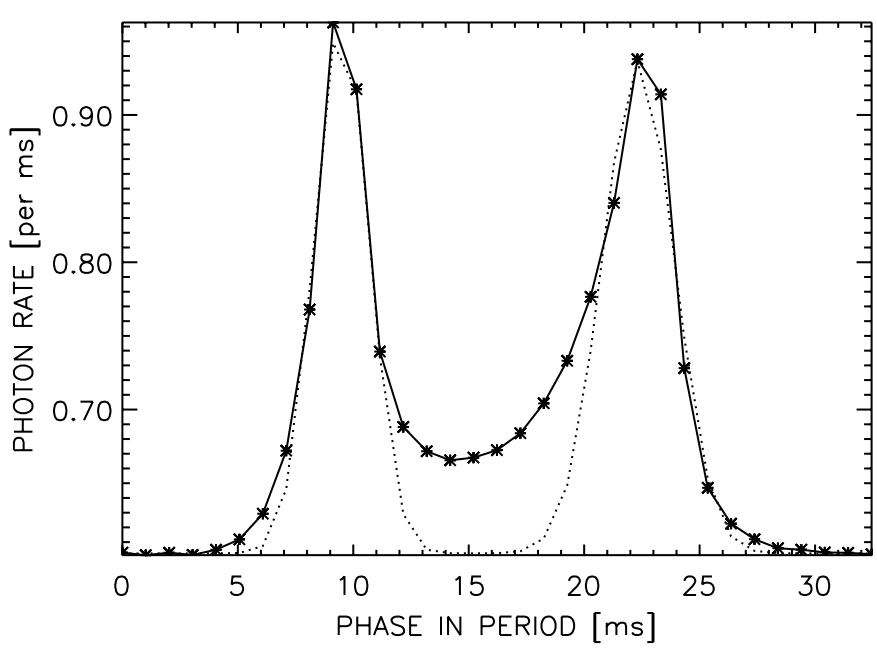

Fig. 1. Integrated profile of Crab pulsar after summing 1868112 periods from 23 files. The abscissa is time (also called phase) within the period (in ms), while the ordinate is the average number of photons obtained in one synthesized time sample $(1.013967 \mathrm{~ms})$. The mis-alignment of the profiles from file to file does not exceed half a synthesized time sample. The dotted curves represent the two peaks modeled as Gaussian.

Time samples having incomplete exposure were deleted. These occurred naturally at the beginning and end of each light curve, and also whenever the RXTE observatory shut off some PCUs, for technical reasons. Each light curve was then converted into the ASCII format for further processing.

The second phase of data analysis used self-developed software. First the power spectrum of each light curve was computed to obtain the period of Crab pulsar in that file. This was used to separate the light curve into individual periods (also called single pulses). Each period has 33 time samples (also called bins), giving a synthesized sampling interval that is different from file to file, but is $\approx 1.013967 \mathrm{~ms}$. The above separation was done such that the photon counts in each original sampling interval were not split across more than one synthesized sampling intervals; otherwise the Poisson statistics of the data would be distorted, and would cause problems for some studies as discussed later on. The separation into individual periods for radio data is much simpler, since standard resampling techniques can be used.

The appropriate period for each data file was ascertained more accurately by checking for "drift" of the integrated profile between the first and second halves of the light curve; this part was done iteratively (see Vivekanand et al. 1998 for details). A straight line fit, to the starting epoch (TDB system) of each data file versus the Crab pulsar period in that file, gives a period derivative of $4.208( \pm 0.005) \times 10^{-13} \mathrm{~s} / \mathrm{s}$, which compares excellently with the actual value for Crab pulsar. The standard deviation of the periods about the fitted straight line is $\approx 2$ nano seconds (ns), which is consistent with the expected value; the data files contain typically 50000 to 98000 periods, and one can recognize a relative shift of a small fraction

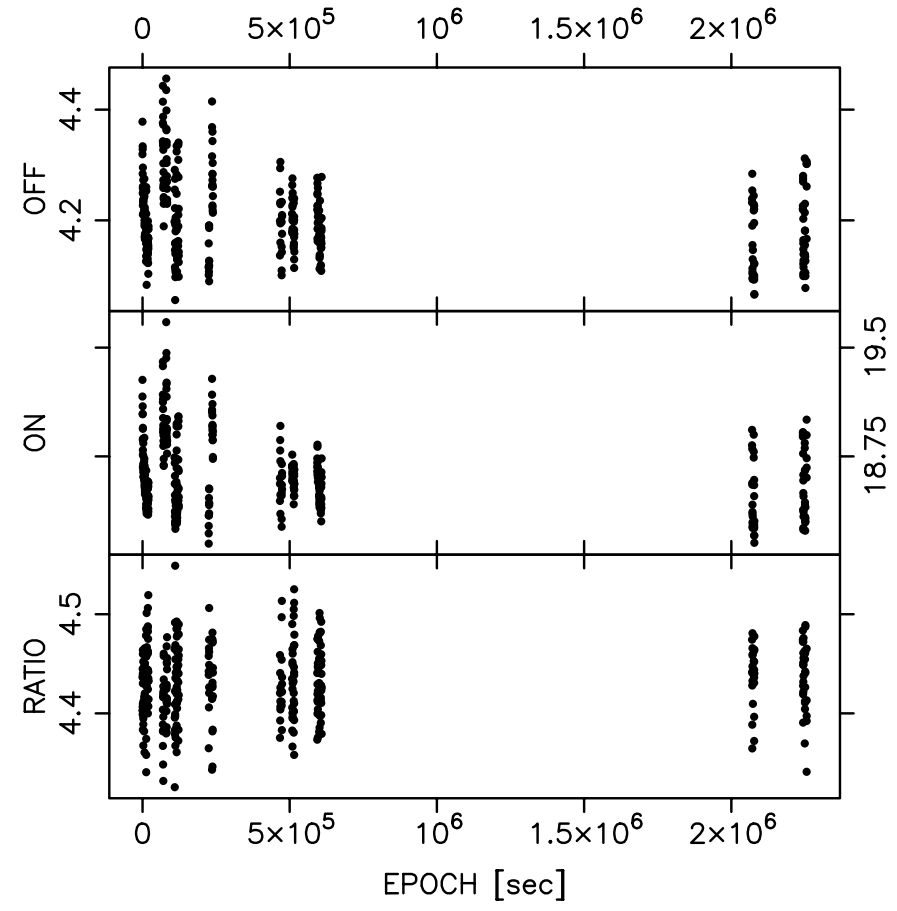

Fig. 2. X-ray light curve of Crab pulsar over 26 days during August/September 1996. The first two plots show the average number of photons in the off-pulse window (OFF) and onpulse window $(\mathrm{ON})$ defined in Fig. 1. The last plot is the ratio $\mathrm{ON} / \mathrm{OFF}$.

of the time sample between the integrated profiles of the first and second halves of a data file. However the periods differ systematically by $\approx 8$ ns from those obtained using Crab pulsar's ephemeris. It is not clear to this author why this systematic difference should occur, but this does not affect the rest of the analysis.

Figure 1 shows the integrated profile of Crab pulsar for 1868112 periods. The integrated profiles of all files are aligned, correct to half a time sample, so that one can analyze them as if all 23 files have been obtained "in phase". However it does smear the integrated profile to a maximum of half a synthesized time sample. Samples 5 to 30 are considered to represent the on-pulse window, and the rest of the seven samples the off-pulse window, although the Crab pulsar might emit X-rays all through its period.

Details of the analysis in the coming sections can be found in Vivekanand \& Joshi (1997), Vivekanand et al. (1998), and Vivekanand (2000); they will be described only briefly in this article.

\section{X-ray light curve}

Figure 2 shows the average number of photons in the offand on- pulse windows of Fig. 1. Each point in the figure represents the average number of photons in 5000 periods $(\approx 167 \mathrm{~s})$; the total duration in the figure is 26.1 days. Both ON and OFF counts show correlated hourly as well as daily variation. Assuming that this is most probably an instrumental effect, the ratio ON/OFF in Fig. 2 would 
represent the true variation of the Crab pulsar X-ray flux. The mean value of the ratio is 4.432 while its standard deviation is 0.035 ; so the $\mathrm{X}$-ray flux of Crab pulsar fluctuates with a standard deviation of $0.035 / 4.432 \approx 0.8 \%$ of its mean value, which is a negligible quantity. This supports the reported Crab pulsar behavior at 0.015 to 0.130 mega electron volt (MeV) energies (Ubertini et al. 1994), but is contrary to its behavior at $\approx 200 \mathrm{MeV}$ reported by Nolan et al. (1993) using CGRO observations, where they found the $\gamma$ ray flux of Crab pulsar to vary over time scales of a month. However the above result $(0.8 \%)$ would be an underestimate if there is significant emission from the Crab pulsar during the off-pulse window.

It would have been interesting to study the existence, at $\mathrm{X}$-rays, of the reported very long time scale (of $\approx 13.5$ years) variation of the ratio of the fluxes in the two peaks (of Fig. 1) at $\gamma$-rays (Nolan et al. 1993); unfortunately this cannot be done due to the limited data span available here.

\section{Pulse to pulse flux variations}

The last section discussed the flux variations of Crab pulsar over time scales of hours and days. The current section discusses flux variations from period to period.

\subsection{Spectrum of flux variations}

Figure 3 shows the so called X-ray fluctuation spectrum of Crab pulsar. At each of the 33 samples (phases) of the integrated profile of Fig. 1, a time series was formed comprising of the X-ray flux as a function of the period number in the data file. This was Fourier transformed in arrays of length $128 \times 1024$ periods. The data were centered in the array and zero padded, and then a Hamming window was applied. To remove long term variations, the data in blocks of 32768 periods were normalized with the mean value of this block (see Ritchings 1976 and Vivekanand \& Joshi 1997 for details). Fourteen data files were chosen that had at least 75000 periods each, totalling to 1347028 periods. Figure 3 shows the power spectrum averaged over the 33 spectra, after normalizing each spectrum with the variance of its time series. A polynomial of the form

$y=a_{0}+a_{1} x+a_{2} x^{2}+a_{3} x^{3}$

was fit to the power spectrum in Fig. 3. the coefficients are $a_{0}=0.999 \pm 0.001, a_{1}=0.003 \pm 0.013, a_{2}=-0.003 \pm 0.059$ and $a_{3}=-0.004 \pm 0.077$. The standard deviation of the power spectrum with respect to the above fitted curve is 0.046 , which is mainly determined by photon noise. It is clear that none of the coefficients are significant except the first.

The upper limit to any possible weak and broad spectral feature that might be hidden in the data can be computed to be $\approx 0.06 \%$ of the total power in the spectrum. Therefore Fig. 3 is consistent with the Crab pulsar having no spectral feature in its pulse to pulse X-ray flux variations.

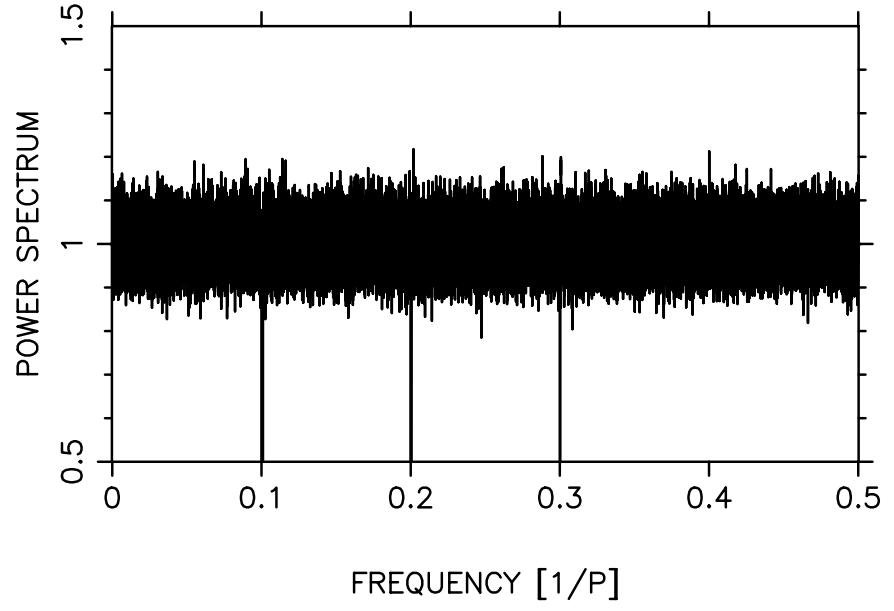

Fig. 3. Average power spectrum of pulse to pulse X-ray flux variations of Crab pulsar. The time series, consisting of X-ray photons as a function of the period number, was Fourier transformed at each of the 33 phases of the integrated profile; the modulus squared of the 33 transforms was averaged. The abscissa in is units of inverse periods, extending up to 0.5 due to the Nyquist criterion. A small range of frequencies has been removed around the sampling spectral feature $(\approx 1 / 10$ periods $)$, and two of its harmonics.

\subsection{Modulation index}

The radio flux of rotation powered pulsars varies significantly from pulse to pulse; this could be due to intrinsic flux variation of the sub pulse, as well as random position of the sub pulse within the on-pulse window (here one is ignoring the flux variations due to propagation in the interstellar medium). This is characterized by the so called modulation index $\mu$, defined as

$\mu=\frac{\sigma_{I}}{\langle I\rangle}$

where $\langle I\rangle$ and $\sigma_{I}$ are the mean and the standard deviation of the pulsar flux (see Manchester \& Taylor 1977); $\mu$ represents the fractional flux variation of the pulsar, and is usually greater than 1.0 at radio wavelengths.

Figure 4 shows the square of $\mu$, which is the natural quantity to average, for all 1868112 periods. First, the mean $\langle I\rangle$ and the variance $\sigma^{2}$ are computed at each sample in the integrated profile $(\langle I\rangle$ is plotted in Fig. 1 and the top panel of Fig. 4). Now $\sigma^{2}$ has contribution from two sources:

$$
\begin{aligned}
\sigma^{2} & =\sigma_{K}^{2}+\sigma_{I}^{2} \\
& =\langle I\rangle+\sigma_{I}^{2}
\end{aligned}
$$

where $\sigma_{K}^{2}$ is the variance due to Poisson statistics of photons, and equals the mean number of photons $\langle I\rangle$, while $\sigma_{I}^{2}$ represents the fluctuation of the average intensity of the pulsar; the two are referred to as photon noise and wave noise, respectively (Goodman 1985). The modulation index squared in Fig. 4 was estimated by subtracting the mean intensity $\langle I\rangle$ from the estimated variance $\sigma^{2}$, and then dividing by $\langle I\rangle^{2}$ at each sample of the integrated profile. Because of the PCA dead time correction to the 


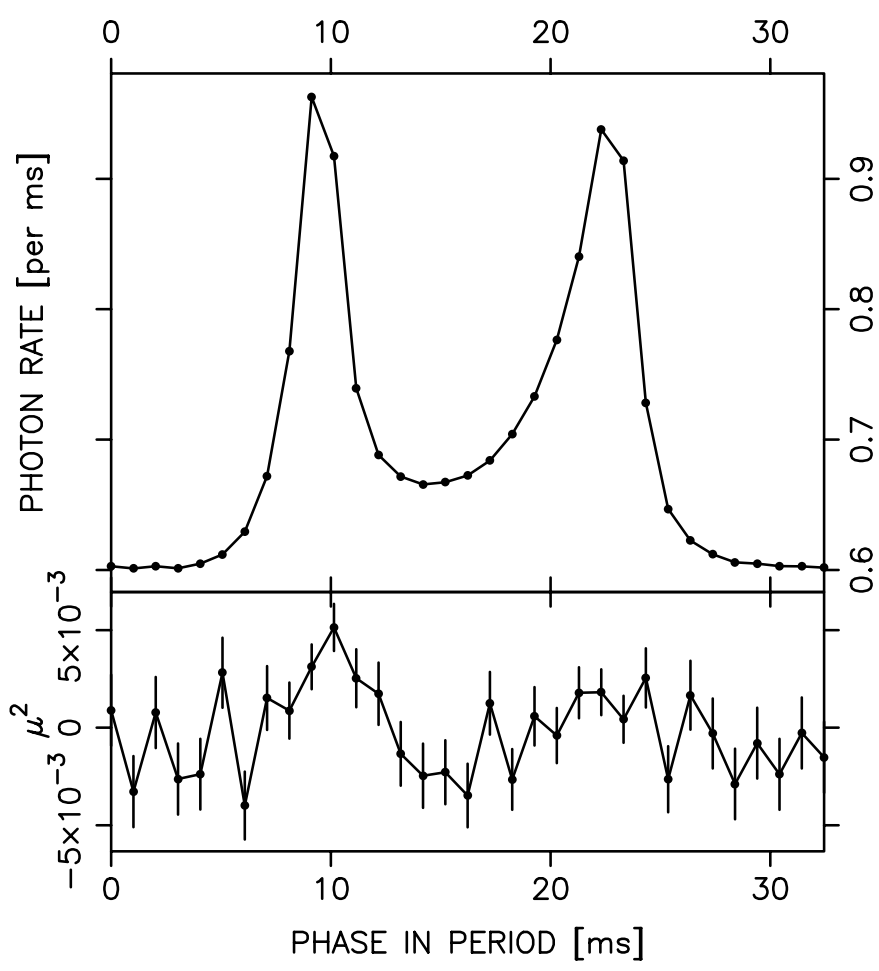

Fig. 4. Square of the estimated modulation index $\left(\mu^{2}\right)$ of the Crab pulsar X-ray flux, at each sample of the integrated profile. The top panel is the same as Fig. 1. The vertical bars represent two standard deviation error bars. Since $\mu^{2}$ depends upon the small difference between two much larger quantities (Eq. (3)), it can be negative also depending upon measurement errors. The distribution of these negative values about the value 0.0 is consistent with their rms errors, as expected.

data, the photon rate of Crab pulsar in any time sample is about $6 \%$ higher than the corresponding integer value of photons. This correction was estimated self-consistently by averaging $\sigma^{2}-\langle I\rangle$ over all 33 samples of the integrated profile, for each file. The average value of the correction for all 23 files is 1.062 with standard deviation of 0.003 . The mean flux $\langle I\rangle$ at each sample was scaled by this constant (for that data file) before subtracting from $\sigma^{2}$. This constant is not dependent upon the average flux $\langle I\rangle$ at each sample, so the above procedure is unlikely to introduce artifacts in the $\mu^{2}$ of Fig. 4.

The average value of $\mu^{2}$ in Fig. 4 is $-0.0002 \pm 0.0040$, which is is consistent with zero as expected. The $\chi^{2}$ of $\mu^{2}$ of Fig. 4 with respect to the expected value of 0.0 is 76.3 for 33 degrees of freedom. Removing sample number 10 reduces the $\chi^{2}$ to 57.0 , and further removing sample number 11 reduces it to 48.7 , which is just 2.25 standard deviations away from the expected value of 31.0. Therefore to the zeroth order of accuracy it is reasonable to assume that $\mu^{2}$ is the same (i.e., 0.0) for all samples in the integrated profile. Its standard deviation is 0.0024 in the on-pulse window; then the standard deviation of $\mu$ can be estimated as $\sqrt{0.0024 / 2} \approx 3.5 \%$ (by simple algebra). Thus the rms X-ray flux variation at any phase in the on-pulse window of the integrated profile of Crab pulsar is $\approx 3.5 \%$ of its mean value. Then the rms variation of the total on-pulse X-ray flux of the Crab pulsar will be $\approx 3.5 / \sqrt{26} \approx 0.7 \%$. A similar calculation for the entire integrated profile gives $\sqrt{0.0023 / 2} / \sqrt{33} \approx 0.6 \%$, which is essentially the same result. This is a much tighter limit than the $\approx 7 \%$ quoted by Patt et al. (1999). These authors used totally 105000 periods and a different method of analysis, on account of which their result might be dominated by photon noise. The result of this section should ideally reflect the actual X-ray intensity variations of Crab pulsar (wave noise), that contain information about the physics of the X-ray emission mechanism.

To the next order of accuracy $\mu^{2}$ appears to be correlated with the shape of the integrated profile; both the lower panel of Fig. 4 as well as the $\chi^{2}$ discussed above point to this. The $\mu^{2}$ at the phase of the second peak of the integrated profile also appears to be enhanced. The on-pulse and off-pulse $\chi^{2}$ are 69.0 and 7.3 , for 26 and 7 degrees of freedom respectively; the former is 6 standard deviations away from the expected value, which is quite high. However more data, or better analysis, is needed to confirm this with good statistical significance.

\subsection{Giant pulses}

Comparison of photon counts in the off- and on-pulse windows of Fig. 1 shows that the Crab pulsar does not emit "giant pulses" at X-ray energies. The mean on-pulse window photon rate is 17.57 photons in 26 time samples, while the maximum is about 31 photons. This implies that at $\mathrm{X}$-ray energies the Crab pulsar emission occasionally increases by $\approx 31.0 / 17.57 \approx 1.76$ times, at the most, whereas at radio wavelengths the giant pulse energy is about 10 to 100 times its mean value (Lundgren et al. 1995). Further, the on-pulse photon distribution fits a Poisson distribution very well, and there is no discernible excess probability at higher photon rates. Finally, following the method of Ritchings (1976) and Vivekanand (1995), a deconvolved photon distribution was obtained that represents the true on-pulse photon distribution of the Crab pulsar; it also does not indicate the presence of an excess probability at higher photon rates.

\section{Correlation of flux in the two peaks}

Patt et al. (1999) report that the X-ray flux in the two peaks of the integrated profile of Fig. 1 are uncorrelated. Their integrated profile consists of 16 samples, in which the two peaks are defined as covering phases 1 to 4 plus 15 and 16, and phases 5 to 11, respectively, (Fig. 1 of Patt et al. 1999). They obtain the covariance between the number of photons in these two peaks to be $-7.7 \times 10^{-4}$, which they claim is consistent with zero. In this work the dotted curves of Fig. 1 are the models for the two peaks. The X-ray flux in each period was multiplied by the two curves, to obtain the flux in the two peaks, respectively. A normalized correlation coefficient (in contrast to the covariance of Patt et al. 1999) was computed for these two fluxes, across all 1868112 periods; the result is $3.5( \pm 10.0) \times 10^{-4}$, which 
is consistent with zero. Therefore it is concluded that the X-ray fluxes of Crab pulsar in the two peaks of its integrated profile are indeed uncorrelated.

\section{Discussion}

The X-ray flux variations of Crab pulsar have a standard deviation of $\approx 0.6 \%$ to $0.8 \%$ of its mean value over time scales ranging from a period to almost a month; that this number is similar over such wide time scales may or may not be a coincidence. This is consistent with the Crab pulsar's behavior at optical and UV energies (Percival et al. 1993) and at IR energies (Lundgren et al. 1995). Therefore the Crab pulsar flux variations at higher energies are insignificant compared to those at radio wavelengths, where the modulation index $\mu$ is $\approx 1.0$, most of which apparently comes from the giant pulses (Lundgren et al. 1995). Now, this causes problems for the conjecture that both the radio and the high energy emission are related through a common electric current. If the flux variations of Crab pulsar are due to temporal variation in the number of basic emitters, then the radio flux variations would also have been at the $\approx 0.7 \%$ level. If they are due to temporal variation in the coherence of the basic emitters, then the radio flux variations would have been at the $2 \times 0.7 \approx 1.4 \%$ level, since the intensity of a coherent emission mechanism is proportional to the square of the number of basic emitters. If they are due to temporal variation in the angle of some elementary beams (Lundgren et al. 1995), one would have expected the radio flux variations to be smaller than those at high energy, since the common beams would be larger at the larger wavelengths. One could surely postulate variations in the angle of radio beams only, but that would have to be justified on the basis of some other independent physical mechanism. In summary the difference in the radio and high energy flux variations of Crab pulsar is difficult to explain, if the basic charged emitters at both wavelengths are somehow related.

Another method, of amplifying the very small flux variations of Crab pulsar at high energies to the very large variations at radio wavelengths, would be to somehow use a fraction of the $\approx 10^{7}$ amplification factor of particles in the gaps, due to cascading $\mathrm{e}^{+}-\mathrm{e}^{-}$pair production (Ruderman \& Sutherland 1975; Cheng et al. 1986a, $1986 \mathrm{~b})$. One could probably postulate that the radiation that is emitted by the later generation of charges in the pair cascade process suffers greater variation in its intensity, due to amplification of the variation in the number density of charges. This might also imply that one should see a monotonic increase in the modulation index as one observes at larger wavelengths. More detailed study of Cheng et al. (1986a, 1986b) and Romani \& Yadigaroglu (1995) models is required for a reasonable solution. In any case, the results of this paper set very strong constraints on the explanation for the relative flux variations at the radio and X-ray energies.

The possible correlation of $\mu$ with the integrated profile in Fig. 4, if confirmed in future, might set very strong constraints on the basic emission mechanism of high energy emission from the Crab pulsar. In the framework of Cheng et al. (1986a, 1986b) and Romani \& Yadigaroglu (1995) models, the two peaks of the integrated profile are cusps created by emission from different magnetic field lines, that add in phase along different directions due to relativistic aberration. A well defined relation between the mean flux and its variance at each point in the integrated profile (for example, $\mu$ might vary as $\langle I\rangle^{\alpha}$ ) will be an additional constraint, along with the exact shape $\langle I\rangle$ of the integrated profile, on the X-ray emission mechanism.

Further work on these data is in progress, that studies issues such as (a) verifying if the Crab pulsar shows at $\mathrm{X}$-ray energies the three phenomenon that are often seen in several radio pulsars - "pulse nulling" "systematic sub pulse drifting" and "mode changing"; (b) looking for special behaviour in the X-ray integrated profile at the phase of the radio precursor, which is supposed to be different from the rest of the radio integrated profile; (c) comparison of the peak and the bridge X-ray emission of the Crab pulsar, which might further constrain the models of Cheng et al. (1986a, 1986b), Romani \& Yadigaroglu (1995), and Cheng et al. (2000).

On the theoretical front, it is probably worth exploring the simultaneous modeling of $\langle I\rangle$ and $\sigma_{I}^{2}$ (or $\mu$ ) at X-rays for rotation powered pulsars, and more specifically for the Crab pulsar.

Acknowledgements. This research has made use of (a) High Energy Astrophysics Science Archive Research Center's (HEASARC) facilities such as their public data archive, and their FTOOLS software, and (b) NASA's Astrophysics Data System (ADS) Bibliographic Services. The author is thankful to them for their excellent services.

\section{References}

Cheng, K. S., Ho, C., \& Ruderman, M. 1986a, ApJ, 300, 500 Cheng, K. S., Ho, C., \& Ruderman, M. 1986b, ApJ, 300, 522

Cheng, K. S., Ruderman, M., \& Zhang, L. 2000, ApJ, 537, 964

Goodman, J. W. 1985, Statistical Optics (New York: Wiley Interscience)

Kawai, N., Okayasu, R., Brinkmann, W., et al. 1991, ApJ, 383, L65

Lundgren, S. C., Cordes, J. M., Ulmer, M., et al. 1995, ApJ, 453,433

Manchester, R. N., \& Taylor, J. H. 1977, Pulsars (San Fransisco: Freeman)

Moffet, D. A., \& Hankins, T. H. 1996, ApJ, 468, 779

Nolan, P. L., et al. 1993, ApJ, 409, 697

Patt, B. L., Ulmer, M. P., Zhang, W., Cordes, J. M., \& Arzoumanian, A. 1999, ApJ, 522, 440

Percival, J. W., et al. 1993, ApJ, 407, 276

Ritchings, R. T. 1976, MNRAS, 176, 249

Romani, R., \& Yadigaroglu, I.-A. 1995, ApJ, 438, 314

Ruderman, M. A., \& Sutherland, P. G. 1975, ApJ, 196, 51

Ubertini, P., et al. 1994, ApJ, 421, 269

Vivekanand, M. 1995, MNRAS, 274, 785

Vivekanand, M. 2000, ApJ, 543, 979

Vivekanand, M., \& Joshi, B. C. 1997, ApJ, 477, 431

Vivekanand, M., Ables, J. G., \& McConnell, D. 1998, ApJ, 501,823 